\title{
Perspective
}

PERSPECTIVE Actualité en histoire de l'art

2| 2007

La Grande-Bretagne/Période moderne

\section{Choix de publications}

\section{Perspective}

\section{OpenEdition}

\section{Journals}

Édition électronique

URL : http://journals.openedition.org/perspective/3857

DOI : 10.4000/perspective.3857

ISSN : 2269-7721

Éditeur

Institut national d'histoire de l'art

Édition imprimée

Date de publication : 30 juin 2007

Pagination : 401-405

ISSN : 1777-7852

\section{Référence électronique}

Perspective, "Choix de publications », Perspective [En ligne], 2 | 2007, mis en ligne le 23 décembre 2013, consulté le 01 octobre 2020. URL : http://journals.openedition.org/perspective/3857 ; DOI : https://doi.org/10.4000/perspective.3857

Ce document a été généré automatiquement le 1 octobre 2020. 


\title{
Choix de publications
}

\author{
Perspective
}

- Art Historian Information from Central Europe - AHICE, www.ahice.net

Une initiative du Centre international de la culture à Cracovie, de la Galerie de Moravie (République tchèque), de la Faculté d'histoire de l'art de l'Université de Komen (Slovaquie) et du Bureau de la protection du patrimoine culturel (Hongrie) a débouché il y a trois ans sur la création de ce site internet. AHICE est un service d'information et un guide en ligne comprenant le calendrier des événements culturels, en particulier des expositions [J. Chroscicki].

2 - Daniele Benati, Eugenio Riccomini éd., Annibale Carracci, (cat. expo., Bologne, Museo Archeologico, 2006/Rome, Chiostro del Bramante, 2007), Milan, Electa, 2006.

Décevante en raison du trop grand nombre des problèmes d'attributions, cette exposition n'en a pas moins fourni un état stimulant des recherches sur l'artiste [S. Loire].

- Geneviève BRESC-BAUTIER, Françoise BARON, Pierre-Yves LE POGAM éd., La sculpture en Occident. Études offertes à Jean-René Gaborit, Dijon, Éditions Faton, 2007.

Volume de mélanges réalisé en hommage à Jean-René Gaborit, conservateur au département des sculptures du Louvre pendant quarante ans. Les textes rassemblés rendent compte de son inépuisable curiosité intellectuelle qui couvrait tous les champs de la sculpture, du Moyen Âge au XIX ${ }^{e}$ siècle, sans oublier le domaine de l'histoire des collections. Une bibliographie donnant la liste de ses travaux jusqu'en 2005 clôt l'ouvrage [G. Scherf]. 
4 - Stéfanie BuchenAu, Élisabeth DÉcultot éd., «Esthétiques de l'Aufklärung », dans Revue germanique internationale, 2006/4, $224 \mathrm{p}$.

Édité par les éditions du CNRS, ce numéro rassemble les quatorze contributions du colloque qui s'est tenu à l'ENS en 2005 : l'occasion de (re-)découvrir la richesse de la pensée esthétique allemande pré-kantienne illustrée par Baumgarten mais aussi par Bodmer, Breitinger, Gottsched, Mendelssohn, Sulzer et Herder [F. Cousinié].

- Marianne cojannot-Le blanc, Marisa dalai emiliani, Pascal dubouRg-GLATIGNy éd., L'artiste et l'œuvre à l'épreuve de la perspective, (collection de l'École française de Rome, 364), Rome, 2006 [C. Michel].

Les actes de ce colloque dressent un utile panorama européen des différents modes d'utilisation de la perspective et d'élaboration de son savoir en Europe, de la Russie de Pierre le Grand à la cour portuguaise du XvIII ${ }^{e}$ siècle, de Fra Angelico à l'atticisme parisien en passant par les Kunstbücher allemands du XVI ${ }^{\mathrm{e}}$ siècle. Il a en outre le mérite de proposer, pour chacune des grandes parties (élaboration et diffusion du savoir; transmission et mise en œuvre ; constructions historiques) de très utiles introductions qui permettent, par un bref état de la recherche, de mieux comprendre les apports des différentes communications [O. Bonfait].

- Isabelle DE CONIHOUT, Patrick MICHEL, Christian PÉLIGRY éd., Mazarin, les lettres et les arts, (colloque, Paris, 2002), Saint-Rémy-en-l'eau, Bibliothèque Mazarine, 2006 [C. Michel].

Cet important ouvrage consacré à Mazarin collectionneur et mécène contient des études variées sur son activité de mécène et de collectionneur (antiques, livres, objets d'art, tableaux); elles portent également sur l'iconographie du cardinal et son historiographie [s. Loire].

7 - Philip conisBeE éd., French Genre Painting in the Eighteenth Century, (colloque, Washington, 2003), New Haven/Londres, Yale University Press, 2007 [C. Michel].

Ces actes du colloque organisé en décembre 2003 au CASVA à l'occasion de l'exposition The Age of Watteau, Chardin, Fragonard, permettent de dresser un état des approches actuelles de la peinture de genre. La plupart des auteurs sont anglo-saxons, de Susan Siegfied à Ewa Lajer-Burcharth, de Bernadette Fort à Michael Fried, et insistent sur des approches sexuées des thématiques de la peinture de genre. D'autres questions sont évoquées, comme les rapports avec l'Italie (Richard Wrigley), la réception de cette 
peinture (Christian Michel) et le marché de la gravure de genre (Kristel Smentek), ou les ambiguïtés des relations avec le genre du portrait (Philippe Bordes) [0. Bonfait].

- Philippe costamagna, Olivier Bonfait, Monica PReti-hamard, Le goût pour la peinture italienne autour de 1800. Prédécesseurs, modèles et concurrents du cardinal Fesch, (colloque, Ajaccio, 2005), Ajaccio, Musée Fesch, 2006.

Très riche publication qui ne se contente pas d'apporter de nombreuses nouveautés sur la collection Fesch, mais présente le goût de plusieurs Napoléonides et d'une vaste palette d'amateurs européens ou français - du prince au curé de province -, ainsi que la situation romaine au début $\mathrm{du} \mathrm{XIX}^{\mathrm{e}}$ siècle et la réception en France de la peinture italienne, du XVII ${ }^{\mathrm{e}}$ au début du xx $\mathrm{x}^{\mathrm{e}}$ siècle. Avec, en prime, un scoop dû à Pauline PrévostMarcilhacy: un album de lavis représentant des tableaux Fesch, dédié à Betty de Rothschild et dessiné en partie par sa fille Charlotte, qui dut le réaliser entre les deux catalogues de la vente, de 1844 et 1845 [Ph. Sénéchal].

9 - Nicholas CRONK, Kris PEETERS éd., Le comte de Caylus. Les arts et les lettres, (colloque, Anvers, 2000), Amsterdam/New York, Rodopi, 2004.

Ce très riche volume consacré au comte de Caylus figure à présent comme une étape fondamentale dans la redécouverte de cette personnalité de premier plan du siècle des Lumières, renouvelant l'ouvrage excellent mais vieilli de Samuel Rocheblave (1889) et complétant les travaux de Thomas Gaehtgens et Marc Fumaroli. Le recueil est divisé en trois grands ensembles. Le premier, précédé par une étude lumineuse de René Démoris ("Caylus entre théorie et critique d'art»), est consacré aux "perspectives esthétiques ", comprenant notamment de passionnants rapprochements avec Diderot (par Didier Masseau) et Winckelmann (par Élisabeth Décultot), un examen de «l'archéologie comme projet esthétique " (par Julie Boch) et une évocation de «la Rome de Caylus et l'idea del bello » (par Letizia Norci Cagiano). Le deuxième ensemble traite de Caylus et la littérature ("théâtre de société », "contes et badinages»). La dernière partie est une remarquable et déjà indispensable bibliographie critique du comte de Caylus, établie par Kris Peeters et couvrant avec l'index une centaine de pages [G. Scherf].

- Jérôme DELAPLANCHE, Joseph Parrocel, 1646-1704. La nostalgie de l'héroïsme, Paris, Arthena, 2006.

Les monographies Arthena sont toujours solides et remarquablement illustrées (même si parfois un peu compassées dans une approche du type la vie/l'œuvre) et, par l'érudition qui s'y déploie, elles sont remarquablement utiles. Celle-ci se distingue non seulement par la découverte, peu avant la parution, d'un important cycle de Parrocel 
aux Invalides, mais surtout par une intéressante volonté de la part de l'auteur de ne pas dissocier l'analyse de l'œuvre d'une réflexion sur le style - avec notamment la problèmatique du pittoresco - et sur le genre (des batailles, ou celui, plus ambigu, des chasses) [0. Bonfait].

11 - Ralph DEKonincK, Agnès GUIDERDONI-BRusLÉ, 'Emblemata Sacra'. Rhétorique et herméneutique du discours sacré dans la littérature en images, Turnhout, Brepols, 2007.

Une publication de référence sur la littérature spirituelle, l'image et l'emblème. Près d'une quarantaine de contributions qui résultent du colloque organisé à Louvain en 2005 : questions d'histoire et de méthodes, exégèse de l'écriture et de la Création, l'image in absentia, rhétorique et poétique de l'image, le spectacle de l'image, la circulation des images entre sensibilités religieuses, l'efficace de l'image [F. Cousinié].

12 - Pascale DUBus, L'art et la mort. Réflexions sur les pouvoirs de la peinture à la Renaissance, Paris, CNRS éditions, 2006.

Brève et claire étude, nourrie de références aux théories artistiques italiennes, portant sur un thème cher aux historiens des mentalités [F. Cousinié].

13 - Georges FARHAT éd., André Le Nôtre. Fragments d'un paysage culturel. Institutions, arts, sciences et techniques, Sceaux, musée de l'Ile-de-France, 2006.

Superbe ouvrage et exemple réussi d'approche interdisciplinaire d'un grand artiste [F. Cousinié].

14 - Guillaume faroult, Sophie eloy éd., La collection La Caze. Chefs-d'œuvre des peintures des XVII et XVIII ${ }^{e}$ siècles du musée du Louvre, Paris, Musée du Louvre, 2007.

Publié à l'occasion d'une exposition présenté au musée du Louvre et comportant le catalogue sur CD-ROM des 583 peintures offertes au Louvre par Louis La Caze en 1869, cet ouvrage ouvre aussi des perspectives très larges sur l'histoire des collections, celle des institutions artistiques et sur l'état du marché de l'art en France au milieu du XIX ${ }^{\mathrm{e}}$ siècle [S. Loire]. 
15 der Heyde, Bassano del Grappa, Istituto di Ricerca per glis studi su Canova e Il Neoclassicismo, 2006 [G. Scherf].

16 - Thomas W. GAeHTGENS, Nicolas Hochner éd., L'image du roi de François $I^{e r}$ à Louis XIV, Paris, Maison des sciences de l'homme, 2006 [F. Cousinié].

17 - Pierre GÉAL, La naissance des musées d'art en Espagne (XVIII-XIX ${ }^{e}$ siècles), Madrid, Casa de Velazquez, 2005.

Issu d'une thèse de Paris IV dirigée par le regretté Carlos Serrano, ce livre fondamental commence par une présentation du patrimoine artistique dans l'Espagne des Lumières pour reconstruire ensuite l'histoire institutionnelle des musées espagnols entre 1808 et 1868 et terminer par une étude du rôle des musées dans la constitution de la culture artistique [K. Pomian].

18 - Adelheid GEALT, George Knox éd., Domenico Tiepolo. A New Testament, (cat. expo., New York, Frick Collection, 2006-2007), Bloomington, Indiana University Press, 2006 [S. Loire].

19 - Pierre GEORGEL éd., Orangerie, 1934. Les " Peintres de la réalité », (cat. expo., Paris, musée de l'Orangerie, 2006-2007), Paris, Réunion des Musées Nationaux, 2006.

Importante reconstitution d'une exposition fondatrice pour l'histoire de la peinture française du XVII siècle [S. Loire].

20 - Guido GUERzonI, Apollo e Vulcano. I mercati artistici in Italia (1400-1700), Venise, Marsilio, 2006.

Cet ouvrage capital, dû a un historien de l'économie, remet en question un nombre considérable d'idées recues sur les marchés artistiques en Italie du XV au XVIII ${ }^{\mathrm{e}}$ siecle. Il offre des chapitres méthodologiques tres stimulants et, en partant de l'exemple des cours des Este, offre un regard nouveau sur les mécanismes de l'offre et sur la place des artistes dans la société. La derniere section déborde de ce cadre chronologique pour évoquer les mécanismes de protection du patrimoine et l'exportation des oeuvres d'art au XIX ${ }^{\mathrm{e}}$ siècle [Ph. Sénéchal]

Perspective, 2 | 2007 
21 - Michèle-Caroline HECK, Théories et pratiques de la peinture. Sandrart et la 'Teutsche Academie', Paris, Maison des sciences de l'homme, 2006.

La monographie attendue sur l'un des principaux historiens de l'art du $\mathrm{XVII}^{\mathrm{e}}$ siècle nordique [F. Cousinié].

22 - Maria KAŁAMAJSKA-SAEED, Genealogia przez obrazy. Barokowa ikonografia rodu Sapiehów na tle staropolskich galerii portretowych, Varsovie, Instytut Sztuki PAN, 2006.

Travail sur les portraits d'une branche de la famille lituanienne (Sapieha), peints vers 1730 pour le mausolée de Kodeń. Actuellement seuls 64 portraits sont conservés au Château de Wawel (Cracovie). L'auteur compare les portraits existants avec l'iconographie de cette famille (en particulier du XVII ${ }^{\mathrm{e}}$ siècle) et fait le lien avec les autres galeries de portraits en Pologne du XVI ${ }^{\mathrm{e}}$ au XVIII ${ }^{\mathrm{e}}$ siècle [J. Chroscicki].

23 - Lola KANTOR-KAZOVSKY, Piranesi as Interpreter of Roman Architecture and the Origins of his Intellectual World, Florence, L.S. Olschki, 2006.

Ce livre explicite la pensée historique et esthétique de Piranèse telle qu'elle s'affirme dans Le Antichità Romane, Della Magnificenza ed Architettura de' Romani e Parere sul Architettura, et reconstitue les débats suscités par ces travaux. Une contribution importante à la connaissance des controverses sur l'architecture ancienne dans la deuxième moitié du XVIII ${ }^{\mathrm{e}}$ siècle [K. Pomian].

24 - Grażyna KOBRZENIECKA-SIKORSKA, Wizerunki carów rosyjskich. Między ikona a portretem, Olsztyn, Presses Universitaires, 2007.

L'auteur étudie des portraits de tsars depuis Ivan IV le Terrible jusqu'à Nicolas II sur fond de tradition d'icônes, mettant en évidence leur sacralisation, les changements stylistiques et les liens avec le portrait royal et nobiliaire polonais de l'époque baroque. Longs résumés en russe et en anglais [J. Chroscicki].

- Stefania LAPENTA, Raffaella MORSELLI, Le collezioni Gonzaga. La quadreria nell'elenco dei beni del 1626-1627, Milan, Silvana, 2006.

Concluant une série de volumes de documents consacrés au collectionnisme de la famille Gonzague aux $\mathrm{XVI}^{\mathrm{e}}$ et $\mathrm{XVII}^{\mathrm{e}}$ siècles publiée depuis 2000 et comptant déjà dix 
autres titres, celui-ci offre un catalogue complet des tableaux des collections Gonzague avant leur dispersion en 1627 [S. Loire].

- Sylvain LAVEISSIÈRE, Léna WIDERKEHR, Jacques Stella (1596-1657), (cat. expo., Lyon, Musée des beaux-arts, 2006-2007/Toulouse, Musée des augustins, 2007), Paris, Somogy, 2006 [S. Loire].

- Ulrich LEBEN, L'École royale gratuite de dessin de Paris (1767-1815), Saint-Rémy-en-l'Eau, Monelle Hayot, 2004.

Passionnante étude sur cet établissement fondé et dirigé par Jean-Jacques Bachelier. Ulrich Leben a utilisé de nombreux documents d'archives inédits ou oubliés pour faire revivre cet établissement (bâtiments, corps professoral, organisation pédagogique) en le plaçant dans le contexte général de l'enseignement du dessin à Paris. Une cinquantaine de pages d'annexes, et des notes nourries rendent cet ouvrage indispensable aux historiens [G. Scherf]. Cet ouvrage fera prochainement l'objet d'un compte rendu dans Perspective.

Description précise des joyaux de portraits des souverains polonais et de leurs familles du XVI $I^{e}$ siècle à la fin du XVII ${ }^{e}$ siècle. L'auteur traite aussi des rares exemples des joyaux royaux conservés dans les collections polonaises et étrangères et évoque le rôle des joyaux dans la société de l'époque (bijoux féminins, insignes funéraires, objets de dévotions etc.). Un riche dictionnaire terminologique de 160 pages établi à partir des sources historiques complète l'ouvrage [J. Chroscicki].

- Stéphane LoIRE, Peintures italiennes du XVII siècle du musée du Louvre. Florence, Gênes, Lombardie, Naples, Rome et Venise, Paris, Musée du Louvre/Gallimard, 2006.

Après un premier volume du même auteur consacré à l'école de Bologne (1996), cet ouvrage complète la publication du catalogue raisonné des peintures italiennes du XVII ${ }^{\mathrm{e}}$ siècle du musée du Louvre. Avec plus de deux cent quatre-vingt œuvres, cet ensemble de peintures baroques est sans doute l'un des plus riches et des plus variés que l'on puisse voir en dehors d'Italie. A la différence du premier volume, la plupart des tableaux sont ici reproduits en couleurs, mais les notices sont toujours aussi fouillées et complètes [0. Bonfait]. 
- Margaretta M. LOVELL, Art in a Season of Revolution. Painters, Artisans, and Patrons in Early America, Philadelphie, University of Pennsylvania Press, 2005.

Ce livre solidement documenté sur la culture dans les villes de la Nouvelle Angleterre $\mathrm{au} \mathrm{XVIII}{ }^{\mathrm{e}}$ siècle est focalisé sur la fabrication et la consommation de produits rendus attrayants par leur valeur artistique. Inversant la démarche habituelle, l'auteur fonde ses analyses sur la reconnaissance de l'esprit artisanal qui caractérise alors la pratique des artistes, même celle des portraitistes les plus en vue. Constatant que la plupart des études mettent l'accent sur le génie individuel des créateurs et sur les choix éclairés de leurs commanditaires, elle propose plutôt la vie familiale comme facteur déterminant de son analyse de la période coloniale, en invoquant à la fois la nature des relations dans l'atelier et l'espace de réception domestique en l'absence de lieux d'expositions et de vente. Hors de la cellule familiale dont il fallait sans cesse négocier de subtiles relations d'hiérarchie et d'interdépendance, c'est l'activité commerciale qui offrait une ouverture sur le monde. Toutefois, l'historien doit constater que les contemporains sont peu diserts sur cette voie de formation identitaire des artistes, artisans et clients. C'est donc sur un examen attentif des objets qu'il faut compter, non pour vérifier les données que la culture de l'époque est censé fournir, mais au contraire pour comprendre et définir cette culture. La pluralité des réalisations - les séries, les modèles répétés et les genres préférés - révèlent ainsi les besoins et aspirations que partagent les acquéreurs, ici analysés sans les clichés psychologisants auxquels les motivations des collectionneurs sont souvent réduites. Reprenant à son compte le projet de Michael Baxandall, l'auteur s'efforce de voir les objets avec «l'œil de l'époque ». Au cœur d'une démonstration qui aborde de nombreux aspects du sujet et présente de nombreux artistes qui seront peu familiers, les trois chapitres inspirés par l'analyse des œuvres de John Singleton Copley sont particulièrement éloquents. Celui sur l'engouement pour la représentation du groupe familial souligne tous les enjeux d'un genre que l'on pouvait croire étouffé par ses conventions [Ph. Bordes].

31 - Francesca LUI, L'antichità tra scienza e invenzione. Studi su Winckelmann e Clérisseau, Bologne, Minerva, 2006.

L'essentiel du livre étudie Clérisseau et son œuvre dans ses rapports avec Winckelmann, avec les artistes étrangers vivant en Italie dont Robert Adam et les Italiens dont Piranèse. Le dernier chapitre traite de Clérisseau après son retour en France, de ses relations avec Séguier et de ses Antiquités de la France [K. Pomian].

32 - Philippe Malgouyres, Charles Mellin. Un lorrain entre Rome et Naples, (cat. expo., Nancy/ Caen, 2007), Paris, Somogy, 2007.

Ce catalogue d'exposition richement illustré et fort bien documenté permet de faire le point sur une énigme de la peinture française, et d'en dresser un parcours artistique 
relativement cohérent: Charles Mellin, qui fut pendant longtemps la solution alternative pour les tableaux poussinesques que l'on ne pouvait rattacher à Poussin luimême. Philippe Malgouyres réussit, alors qu'en effet l'artiste a un style qui évolue rapidement et se fond parfois dans celui de son entourage, que ce soit l'atelier de Vouet ou le palais Muti, à reconstituer la personnalité artistique du peintre, notamment à travers ses dessins. En plus des notices détaillées des œuvres exposées, l'ouvrage contient un répertoire complet des tableaux et dessins reconnus comme autographes, attribués ou refusés. Une contribution significative, après l'exposition de Strasbourg (Eclairage sur un chef-d'œuvre. Loth et ses filles de Simon Vouet, Strasbourg, Musée des beaux-arts, 2005-2006), à la galaxie Vouet et au panorama artistique de la Rome des Barberini [O. Bonfait].

- Fernando mazzocca, Giuseppe Pavanello, Antonio Canova. La cultura figurativa e letteraria dei grandi centri italiani. 1: Venezia e Roma, (colloque, Bassano del Grappa, 2005), Bassano del Grappa, Istituto di ricerca per gli studi su Canova e il Neoclassicismo, 2005.

34 - Fernando MAZzocca et Gianni VENTURI, Antonio Canova. La cultura figurativa e letteraria dei grandi centri italiani. 2: Milano, Firenze, Napoli, (colloque, Bassano del Grappa, 2006), Bassano del Grappa, 2006.

- Fernando MAzzocca, Manlio PASTORE STOCCHI, La gloria di Canova, (colloque, Bassano del Grappa, 2007), Bassano del Grappa, 2007.

Il convient de saluer les publications érudites et élégantes que l'Istituto di ricerca per gli studi su Canova e il Neoclassicismo de Bassano del Grappa édite avec une impeccable régularité, depuis 1998 pour la collection Testi - neuf volumes déjà parus, le dixième, en cours, consacré à Leopoldo Cicognara -, et depuis 2004 pour la collection Studi - cinq volumes parus (les deux premiers étaient intitulés en 2004 Canova direttore di Musei, et en 2005 Il primato della scultura: fortuna dell'antico, fortuna di Canova, tous deux réalisés sous la direction de Manlio Pastore Stocchi). Antonio Canova est la personnalité centrale autour de laquelle gravitent toutes ces études, la collection Testi s'attachant à rééditer des textes anciens (d'Este, Falier, Teotochi Albrizzi, Missirini, Fernow ...) aujourd'hui introuvables.

Rappelons que les études canoviennes bénéficient d'une autre série de publications, sous l'égide de la Fondazione Canova de Possagno, les Quaderni del Centro Studi Canoviani, une collection dirigée par Giuseppe Pavanello (trois volumes parus: G. Pavanello, Canova collezionista di Tiepolo, 1996; P. Mariuz, Leopoldo Cicognara ad Antonio Canova, 2000 ; M. Pavan, Scritti su Canova e il neoclassicismo, 2003).

Enfin, signalons pour mémoire que l'Istituto Veneto di Scienze, Lettere ed Arti de Venise a publié en 1997 les actes du colloque Antonio Canova, et en 2000 l'imposant 
recueil de textes rassemblés par Giuseppe Pavanello, Antonio Canova e il suo ambiente artistico fra Venezia, Roma e Parigi [G. Scherf].

- Philippe MOREL éd., L'art de la Renaissance entre science et magie, (colloque, Rome, 2006), Rome/Paris, Académie de France à Rome/Somogy, 2007.

Un ouvrage qui ouvre des champs et des perspectives nouveaux [C. Michel]. Remarquable synthèse sur l'un des thèmes de recherche les plus originaux de la Renaissance, éditée dans la belle Collection d'histoire de l'art de la Villa Médicis : l'astrologie et ses images, les astres et la religion, astrologie et politique, alchimie et cabale, la nature et ses doubles, magie, talisman et divination [F. Cousinié].

- Stanisław mossaKowsKI, Kaplica Zygmuntowska 1513-1533. Problematyka artystyczna i ideowa mauzoleum króla Zygmunta I, Varsovie, Liber pro arte, 2007.

Monographie fondamentale d'une œuvre d'art du début du XvI siècle en Pologne, après une solide restauration récente. Fruit d'un travail de plusieurs années du professeur Mossakowski, cet ouvrage propose une liste exhaustive des sources en latin et en polonais et une analyse stylistique riche. Une version anglaise de ce livre remarquable est en préparation [J. Chroscicki].

- Jana K.ostRowsKIEGo, Kościoły i klasztory rzymskokatolickie dawnego województwa ruskiego, Cracovie, Centre culturel international, 2006.

Cette série, publiée depuis 1994, est un essai d'inventaire des églises catholiques romaines primitives d'Ukraine, y compris les édifices dispersés, en tenant compte du système administratif d'avant les partages de la Pologne à la fin du XviII ${ }^{e}$ siècle. Le tome 14 inclus entre autres les sanctuaires de Delatyn, Halicz, Kołomyja, Worochta, Žabie. Paralèllement, deux autres séries sont éditées sur les églises de la voïvodie de Vilnius et de Nowogród (Lituanie actuelle) [J. Chroscicki].

- Roberta PANZAelli, Monica PReti-hamard éd., La circulation des ceuvres d'art. The circulation of works of art in the revolutionary era, 1789-1848, (colloque, Paris, INHA/Getty Research Institute, 2004), Rennes, Presses Universitaires de Rennes, 2007.

Actes d'un colloque qui complètent ceux d'une réunion précédente sur les collections et le marché de l'art publiés en 2005 [S. Loire]. 
- Shimpa PRASAD, Guercino. Stylistic Evolution in Focus (cat. expo., San Diego, Timken Museum of Art, 2006-2007), Santa Rosa (CA), Putnam Foundation/University of Washington Press, 2006.

Cette approche nouvelle et bienvenue de l'art du Guerchin, qui convoque aussi bien les sources écrites que l'analyse picturale et la réflexion théorique, se concentre sur trois aspects de son œuvre : l'intérêt pour le théâtre dans le milieu intellectuel de la petite ville de Cento, qui permet à l'auteur de proposer une belle analyse du dessin Une représentation théâtrale à ciel ouvert (British Museum); le "stile rappresentativo ", pour reprendre l'expression de Giovanni Battista Doni, de ses premières peintures, notamment dans la première version de l'Erminie chez les bergers; l'évolution du rôle et de la place du spectateur dans l'œuvre, à travers l'analyse des séries du Fils prodigue ou des autoportraits [0. Bonfait].

41 - Daniel RABREAU, Sandra PASCALIS éd., La nature citadine au siècle des Lumières. Promenades urbaines et villégiature, (colloque, Nancy, 2005), Bordeaux, William Blake \& Co/Art \& Arts, 2005.

Actes du colloque tenu au Musée des beaux-arts de Nancy les 24 et 25 juin 2005, et cinquième et brillant volume des Annales du Centre Ledoux, consacré aux liens entre la nature et la ville, avec un riche éventail de communications aux sujets divers et souvent neufs [G. Scherf].

42 - Sophie RAUX éd., Collectionner dans les Flandres et la France du Nord au XVIII siècle, (colloque, Lille, 2003), Lille, Université de Lille 3, 2005.

Précieux récueil de contributions sur le marché de l'art et les collections dans une aire géographique qui s'étend de Arras à Gand. Une première partie rassemble des monographies sur des collectionneurs lillois (Charles Lenglart, les familles Vandercruisse et Imbert de la Basecque) ou sur des artistes collectionneurs à Bruges et à Gand. Le second ensemble regroupe des contributions sur le marché de l'art à Gand et à Lille, ainsi que sur les échanges commerciaux entre Paris et les Flandres, notamment à travers les marchands du Pont Notre-Dame ou les achats du gouverneur des Pays-Bas autrichiens, Charles de Lorraine. Enfin, une troisième partie, plus convenue dans son contenu, porte sur la place de la peinture nordique dans le marché de l'art et les collections à Paris, mais aussi dans les Pays-Bas autrichiens. La diversité des approches, des études de collection avec la disposition des tableaux aux analyses statistiques, ajoute à l'originalité du cadre géographique retenu [0. Bonfait]. 
43 XIXe siècles, (colloque, Ascona, 2002), Hildesheim/Zurich/New York, Societäts, 2007.

Panorama utile des modes d'approche [C. Michel].

- Guilhem SCHERF, Houdon 1741-1828. Statues, portraits sculptés, Paris, Musée du Louvre/ Somogy, 2006.

Ce catalogue de la collection du Louvre recense quarante neuf pièces, dont quinze classées sous des rubriques secondaires (atelier de, d'après, manière de, anciennement attribué à). L'auteur, conservateur au Louvre, précise avec un talent rare le statut et les qualités de ces œuvres auprès desquelles il travaille quotidiennement. Les quelques témoignages invoqués dans la brève introduction confirment la réputation de premier sculpteur de son temps acquise par Houdon dans les années 1770 et 1780, avant l'entrée en scène de Canova. Le rôle de l'atelier est souligné à propos d'une « production sérielle de qualité ", bien distinguée des plâtres d'édition "réalisés dans des creux usés ou par surmoulage ». Les commentaires sur chacune des œuvres, véritables leçons du regard réclamées par l'art de la sculpture, prennent en compte les étapes et procédures de fabrication, ainsi que les aléas de conservation. Les contextes immédiats de création sont fort bien restitués et détaillés. Il faut espérer que ce travail exemplaire inspirera des études sur l'art et la célébrité de Houdon, qui, à l'exception du récent Essai sur les visages des bustes de Houdon de Willibald Sauerländer, a suscité peu de travaux interprétatifs [Ph. Bordes].

- Anne SURGERS, Et que dit ce silence? La rhétorique du visible, Paris, Presses de la Sorbonne nouvelle, 2007.

Au-delà des discours convenus sur l'importance du modèle rhétorique pour la peinture, une tentative de lecture concrète d'œuvres $\mathrm{du} \mathrm{XVII}^{\mathrm{e}}$ siècle au moyen de quelques figures importantes classées sous la forme, pratique, d'un dictionnaire : anacoluthe, antithèse, apostrophe, chiasme, ellipse, épanorthose, etc. [F. Cousinié].

46 - Jonathan UnGLAUB, Poussin and the Poetics of Painting. Pictorial Narrative and the Legacy of Tasso, New York, Cambridge University Press, 2006.

Utile ouvrage de réflexion et de synthèse sur les multiples relations entre la poétique de Poussin et celle du Tasse. L'auteur ne se contente pas d'étudier les rapprochements entre les deux conceptions de l'art de Poussin et du Tasse (le parallèle entre la peinture et la rhétorique, la notion d'imitation et de «novità ») ou les passages entre le poème et sa représentation picturale, mais il aborde plus généralement la question du langage 
poétique à Rome au début du XVII ${ }^{e}$ siècle et du problème de la narration de l'histoire par les voies de la peinture [0. Bonfait].

47 - The Arts of France from François Ier to Napoléon Ier, (cat. expo., New York, Wildenstein Gallery, 2005), New York, Wildenstein Gallery, 2005.

Comporte le catalogue d'une centaine de peintures et dessins, la plupart de grande qualité, qui rendent compte du rôle essentiel joué depuis la fin du XIX e siècle par l'un des grands acteurs du marché de l'art international pour l'appréciation de l'art français $\mathrm{du} \mathrm{XVI}{ }^{\mathrm{e}}$ au XVIII ${ }^{\mathrm{e}}$ siècle [S. Loire].

- Andrzej wiтKo, Tajemnica Las Meninas. Antologia tekstów, Cracovie, AA, 2006.

L'auteur a choisi de publier en polonais dans une excellente traduction treize interprétations du tableau de Velasquez, classées chronologiquement, parmi lesquelles celles d'Antonio Palomino, Karl Justi, Leo Steinberg, Victor Stoichita, Bernard Dorival, Fernando Marias et Amy M. Schmitter. D'autres, comme par exemple John F. Moffitt, dont il n'a pu obtenir les droits de publication sont commentés dans un essai à la fin du volume. La jaquette du livre rappelle l'interprétation de Kenneth Clark, avec l'échange des places d'honneur entre l'Infante et la naine Mari-Barbola [J. Chroscicki]. 